\title{
Multibody Model of a Motorbike with a Flexible Swingarm
}

\author{
Gianni Ferretti ${ }^{1} \quad$ Bruno Scaglioni $^{2} \quad$ Andrea Rossi $^{2}$ \\ ${ }^{1}$ Politecnico Di Milano, Dipartimento di Elettronica, Informazione e Bioingegneria DEIB \\ Via Ponzio 34/5, 20133 Milano, Italy \\ ${ }^{2}$ MUSP Lab, Via Tirotti 9, Le Mose, 29122 Piacenza, Italy
}

\begin{abstract}
Considering the specific case of the multibody modelling of a racing motorbike, where the rigid model of the rear swingarm has been replaced with a flexible one, a general approach to flexible multibody systems modelling in Modelica is presented in this paper. In particular, the steps required to generate the model of a flexible body starting from a FEM analysis, performed with commercial packages, are detailed. Simulations results are shown with reference to a sudden braking and to a series of impacts with curbs. In this last case, an unstable behaviour occurred when considering the flexible component, which is currently under investigation.

Keywords: motorcycle dynamics; flexible multibody systems; finite element method; floating frame of reference; Craig-Bampton method.
\end{abstract}

\section{Introduction}

Object-oriented modelling, favoring a real modular and multi-domain approach, has been recognized as a fundamental tool for the mechatronic design, requiring an integrated approach to mechanical, electronic and control design [1]. In this respect, even if multibody dynamics is frequently just one of the physical domains involved, the simulation of flexible multibody systems plays an important role.

On the other hand, in real applications, the task of modelling distributed flexibility cannot be addressed without the help of finite elements (FE) codes, in order to describe complex geometries and material properties. Moreover, for the sake of efficiency of numerical simulation, the huge number of nodal coordinates introduced by FE modelling, must be reduced to a much smaller number of modal coordinates, for example through the classical Craig-Bampton method [2].

Two commercial packages exist that pre-process the output of FE codes to get the Modelica model of a flexible body, one has been developed by the German Aerospace Center (DLR) [3], the other has been developed by Claytex Services Ltd.

The DLR FlexibleBodies library provides several Modelica classes, namely a flexible beam model (Beam), an annular plate model (AnnularPlate), a thermoelastic plate (ThermoElasticPlate) and a model for general flexible bodies exported from FE codes (ModalBody). The results of the FE analysis performed by several general purpose codes are first processed by another commercial code: FEMBS, implementing modal reduction in a two-step process. Guyan or Craig-Bampton reduction methods are applied in the first step to keep the flexible body input file to FEMBS small, while in the second step the modes in the frequency range of interest for multibody simulation are selected. The reduced modal representation is then stored in a Standard Input Data (SID) file $[4,5]$, an object-oriented data structure developed to define a standard format to exchange data between FE and MBS codes. When a ModalBody class is instantiated the user has to specify the name of the SID file containing the modal description of the body, which also stores the original mesh of the FE model, used by Dymola to perform the animation of the simulated motion of the flexible body.

The Claytex library generates directly the Modelica model of a flexible body from the output of the model reduction process performed by three FE codes: namely Nastran, Genesis and Abaqus.

Object-oriented modelling of general flexible multibody systems has been also described in [6] (up to the Modelica code), based on the parameters of the flexible body computed by FEM packages, stored in an ASCII file and read by a parsing tool. The model also maintains the efficient choice of the generalized coordinates implemented in the Modelica standard (rigid) multibody library. Thus, when a body is a component of a tree structure, the motion of the local Floating Frame of Reference (FFR) [7] is actually calculated by 
propagating of the kinematic quantities from the root of the tree while, in the case of floating bodies, the body itself is a root, introducing its own generalized coordinates for position and orientation.

In this paper, the above mentioned approach is applied to the multibody model of a racing motorbike, where a flexible model of the rear swingarm has been considered.

First, a rigid multibody model of the motorbike has been developed, with the aim of providing forces and torques applied to the frame and to the swingarm for a following FEM structural analysis, performed for the sake of mechanical design validation. As reference simulation scenarios a sudden braking and a series of impacts with obstacles (curbs) have been considered. Then, the rigid model of the rear swingarm has been replaced with a flexible one and the simulation results have been compared.

In view of the particular simulation experiments considered only limited differences were expected, particularly appreciable during the simulation of impacts. On the other hand, the simulation of the motorbike with a flexible swingarm showed an unstable behaviour in the case of subsequent impacts, largely due to a poor performance of the virtual driver, but undoubtedly induced by swingarm flexibility, since the said behaviour in the rigid case did not occur.

The paper is organized as follows. In Section 2 the rigid multibody model of the motorbike is described, including the simulation scenarios and the related results, moreover, the simulation results are compared to experimental results obtained on the real bike. Section 3 explains the flexible multibody modelling approach used in this paper, describing the adopted theoretical formulation and the most relevant characteristics of the Modelica flexible body model. Further, it describes the procedure which leads to obtain flexible body models in Modelica. In Section 4 the flexible model of the rear swingarm is described, the procedure outlined in the previous section is adopted to obtain a flexible model of the body, then, in Section 5 the previously described simulations are repeated and the results are compared with respect to the rigid model. Section 6 concludes the paper.

\section{Rigid multibody motorbike model}

A rigid multibody model of a racing motorbike, built by RobbyMoto Engineering S.r.L., has been first developed (Fig. 1).

Since the considered scenarios were a sudden brak-

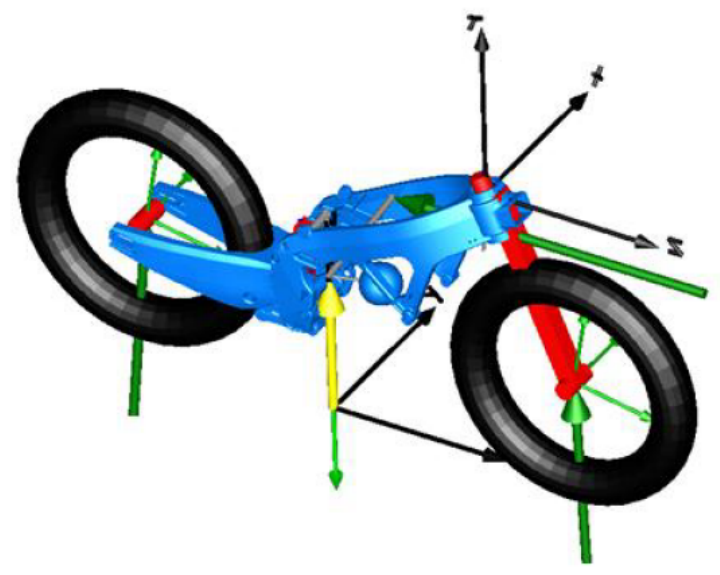

Figure 1: Model of the motorbike.

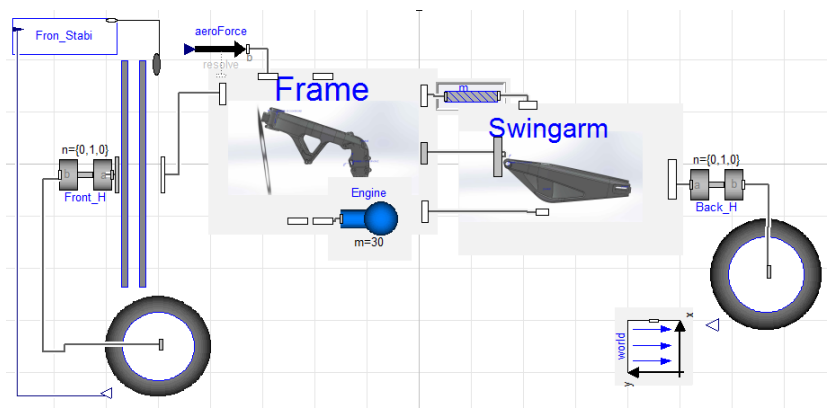

Figure 2: Scheme of the Modelica model of the motorbike.

ing and a series of impacts with obstacles (curbs), the model was not intended to simulate the dynamic behaviour of the motorbike in curves, but a steering degree of freedom was anyway provided, in order to represent the intrinsically unstable behaviour of the vehicle. The adopted tyre model accounted for lateral forces and roll angle, thus a tilt control was required.

The rigid motorbike model is made up entirely by components of the standard Modelica multibody library, except for the wheel/road interaction model and the virtual driver, taken from $[8,9]$. The main components are:

- Main frame,

- Front suspensions,

- Rear suspended Pro-Link swingarm,

- Wheels and wheel-road interaction,

- Virtual driver: a simple vehicle stabilizer, acting on the front steer and controlling the tilt angle.

and the scheme of the Modelica model is shown in Fig. 2. 
It must be pointed out that the suspension mechanism includes two interconnected planar kinematic loops, which must be carefully modelled in order to avoid singularities. In this respect, the Modelica multibody library provides some aggregate joints implementing an analytic solution of the loops closure equations [10]. In our case, a Revolute-Revolute-Revolute (RRR) joint is used to solve one of the loops, as shown in Fig.4, which also inherently breaks the second one, hence there is no need to use another aggregate joint for the second loop. Figure 3 shows a simplified scheme of the planar loop and how it is realised in the real motorbike.
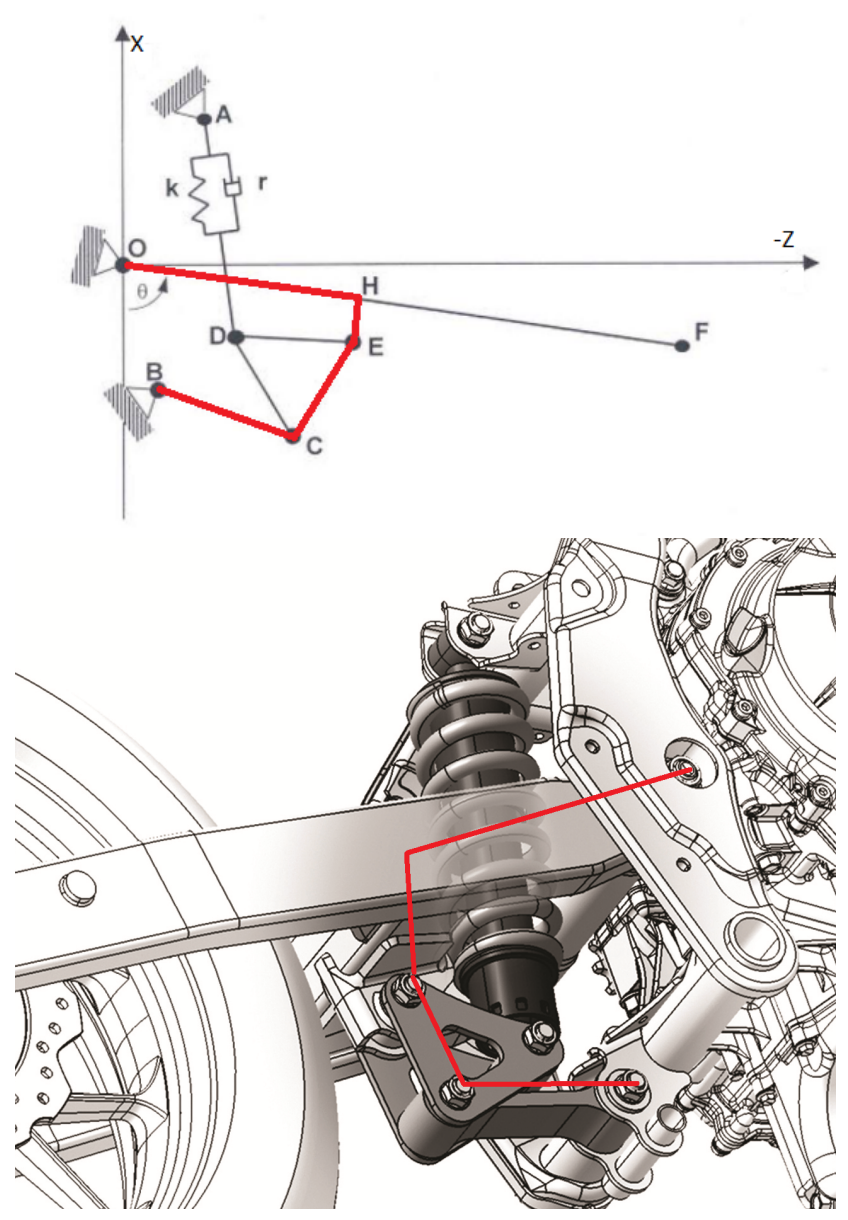

Figure 3: Planar loop.

In view of the simplicity of the considered simulation scenarios, a linear model of the tyre/road interaction has been adopted [8]. The longitudinal force is thus computed as a linear function of the longitudinal wheel slip, and the lateral force is computed as a linear function of the tire sideslip angle and of the roll angle.

Aerodynamic drag forces have been also taken into account, calculated as:

$$
F_{a}=S_{f} C_{z} V^{2}
$$

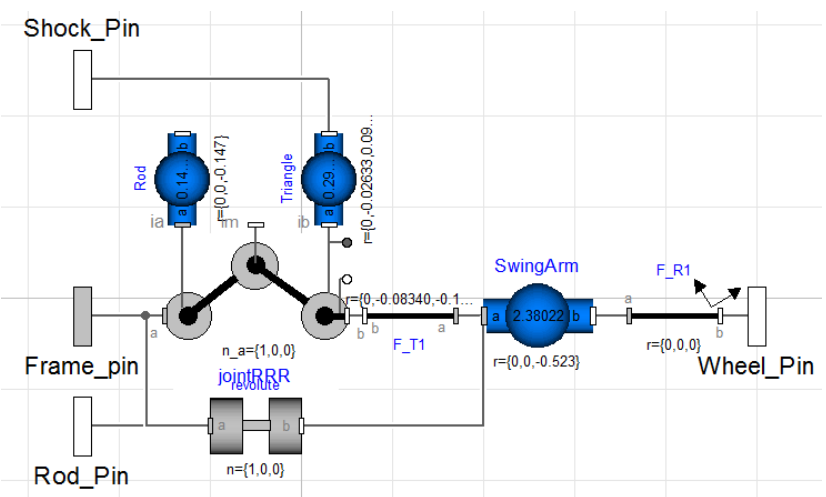

Figure 4: Model of the kinematic planar loop.

Where $F_{a}$ is the aerodynamic force applied in the pressure center of the bike, placed $20 \mathrm{~cm}$ above the center of mass and biased $10 \mathrm{~cm}$ on the front, according to [11]. The front section of the bike is defined as $S_{f}$, the aerodynamic coefficient $C_{z}$ is calculated from experimental results $\left(C_{z}=0.45\right)$, and $V$ is the ground speed of the vehicle. The drag force is shown in Fig. 1 as a green horizontal arrow applied to the pressure center.

\subsection{Simulation of a braking}

In this subsection, the results obtained by simulating a braking are compared to experimental data.

The experiment starts with the bike moving on a straight line at $215 \mathrm{~km} / \mathrm{h}$, then, a constant braking torque of $280 \mathrm{Nm}$ is applied to the front wheel for 4 seconds, according to the pressure observed on the front brake of the real bike. While braking, the pilot weight is applied $70 \%$ on the front handlebars and $30 \%$ on the saddle.

Figure 5 shows two screenshots of the simulation before and during the braking manoeuvre, while Figs. 6 and 7 compare the simulated and experimental speeds and the elongation of the suspensions, respectively. It must be pointed out that the distribution of the driver weight in the Modelica model is constant during the entire simulation, while the distribution of the weight load in the experiment highly depends on the longitudinal acceleration, thus, the front suspension displacement is slightly different between model and experiment before the braking manoeuvre. The comparison between simulation and experiment is more significant in the first part of the transient, say the first 2 seconds, because in the last part the driver enters a curve and starts releasing the brake handle. Anyway, the simulated results appears to be in quite good accordance with the experimental data.

Afterwards, the forces and torques acting on the 


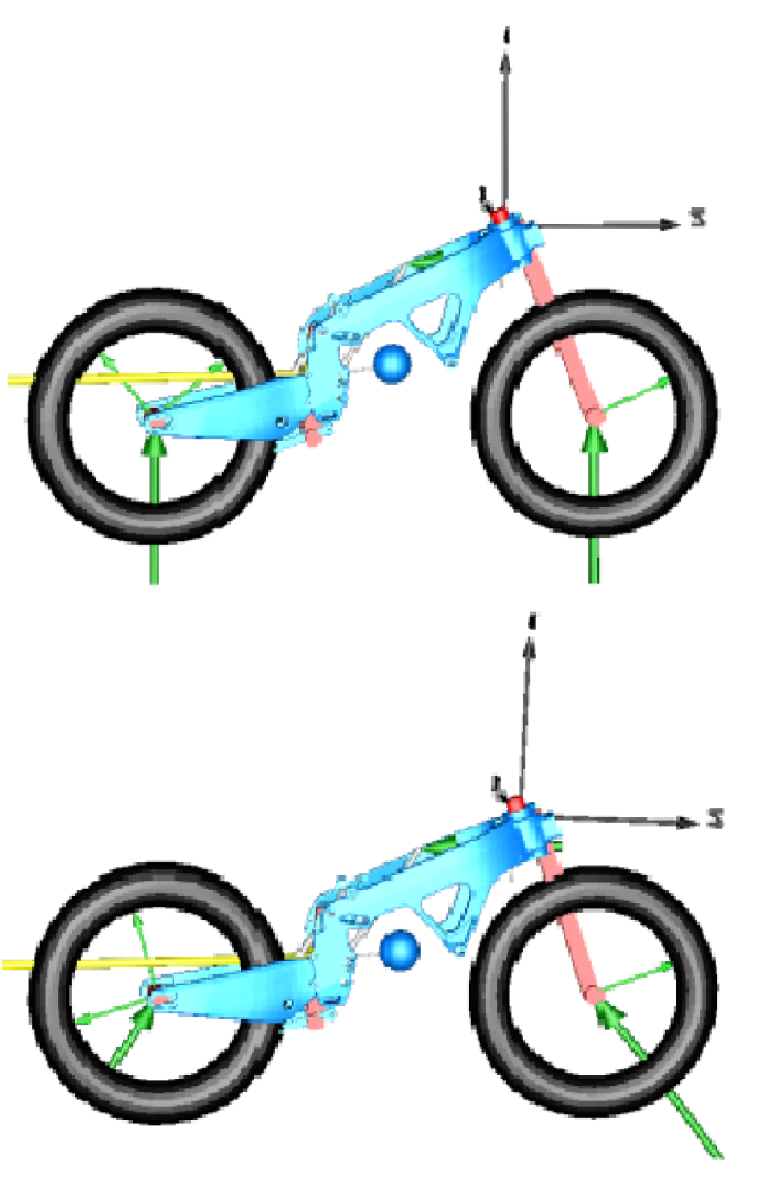

Figure 5: Screenshots of the simulation before and during the braking.

frame and rear swingarm (Fig. 8) have been recorded from the simulation, in order to identify the stress peak and the actual values of loads to be used during a FEM analysis.

\subsection{Impacts with curbs}

In a second simulation experiment a series of impacts with curbs has been considered.

Curbs have been modeled as sawtooth obstacles (Fig. 9) placed on the road surface, every sawtooth has an height of $2 \mathrm{~cm}$ and a width of $20 \mathrm{~cm}$, in order to reproduce the real curbs of most racetracks. To this aim, the road model described in [8] has been modified in order compute the quote of the road, given the position of the wheel. Since the driver weight distribution was impossible to estimate in this experiment, the whole load $(70 \mathrm{~kg})$ was placed on the saddle. The forces and torques acting on the frame and rear swingarm have been exported for a following FEM analysis step, Fig. 10 shows vertical forces exchanged between rear arm and main frame in the hinge.

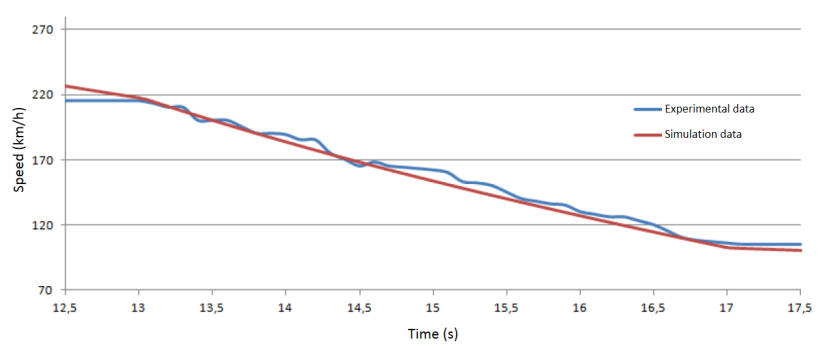

Figure 6: Speeds during braking.

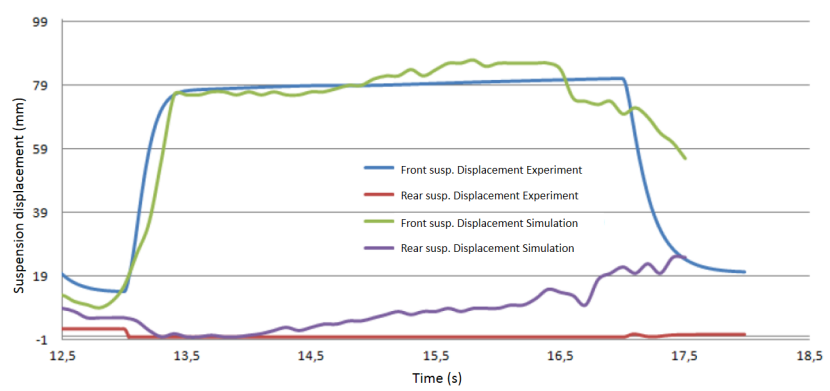

Figure 7: Front and rear suspension elongation during braking.

\section{Flexible multibody modelling in Modelica}

The object-oriented modelling paradigm implemented by the Modelica language requires a description of the dynamics of a flexible body in terms of local variables, while the interaction between different bodies has to be described using the connectors of the standard Modelica multibody library [10]. In turn, a local description of a body's dynamics naturally calls for a floating frame of reference (FFR) approach [7], which is currently the most widely used method in computer simulation of flexible multibody systems.

In the FFR formulation, each body is attached to a moving frame of reference undergoing large (rigid) motion, while the (small) elastic displacements are obtained in local coordinates with respect to the reference frame. Thus, the position (in local coordinates) of a point on a flexible body, see Fig. 11, is given by:

$$
\overline{\mathbf{u}}=\overline{\mathbf{u}}_{0}+\overline{\mathbf{u}}_{f},
$$

where $\overline{\mathbf{u}}_{0}$ is the "undeformed" (i.e., rigid) position vector and $\overline{\mathbf{u}}_{f}$ is the deformation contribution to position (i.e., the deformation field).

If small elastic deflections are considered, according to the classical Rayleigh-Ritz method [12], the infinite dimensional deformation field on the body can 


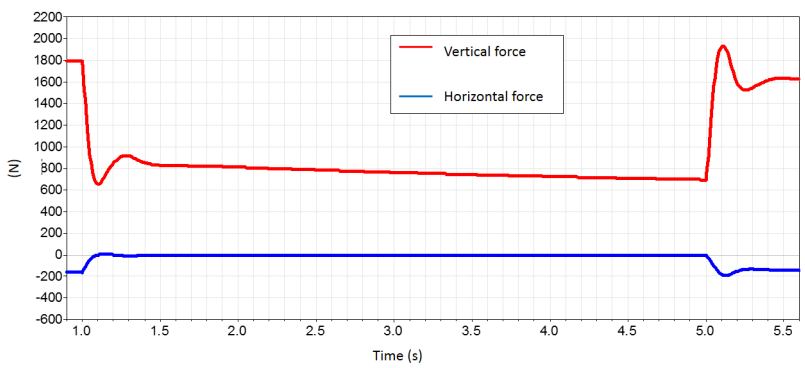

Figure 8: Forces acting on the rear swingarm hinge during braking.

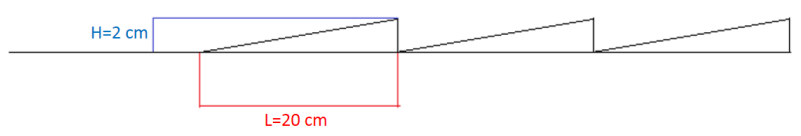

Figure 9: Curbs model.

be approximated by a functional basis space with finite dimension, say $M$, so that the vector $\overline{\mathbf{u}}_{f}$ can be expressed by the finite dimensional product

$$
\overline{\mathbf{u}}_{f}=\mathbf{S q},
$$

where $\mathbf{S}$ is the $[3 \times M]$ shape functions matrix (i.e., a matrix of functions defined over the body domain and used as a basis to describe the deformation field of the body itself) and $\mathbf{q}$ is the $M$-dimensional vector of deformation degrees of freedom, or modal coordinates. The representation of a generic flexible body in the world reference frame requires then $6+M$ d.o.f.: 3 corresponding to the rigid displacements $\mathbf{r}, 3$ to the undeformed body orientation angles $\theta$ and $M$ to the modal coordinates $\mathbf{q}$.

Starting from eqs. $(1,2)$ and accounting for the elastic properties of the material and for the mass distribution, the generalized Newton-Euler equations for a generic unconstrained flexible body, formulated with respect to the FFR, can be derived in $[13,7,14,15,5$, 16], and developed up to the Modelica code in [6]. It must be also pointed out that the efficient choice of the generalized coordinates, implemented in the Modelica standard (rigid) multibody library, can be maintained. Thus, when a body is a component of a tree structure, the motion of the FFR is actually calculated by propagation of the kinematic quantities from the root of the tree while, in the case of floating bodies, the body itself is a root, introducing its own generalized coordinates for position and orientation.

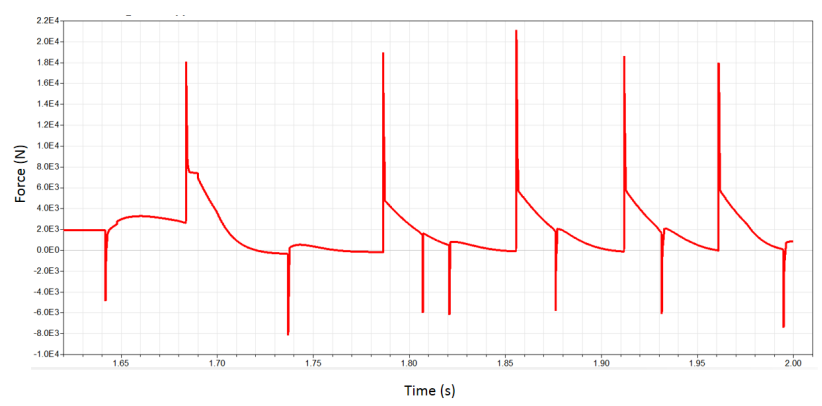

Figure 10: Vertical forces acting on the rear swingarm hinge during impacts with curbs.

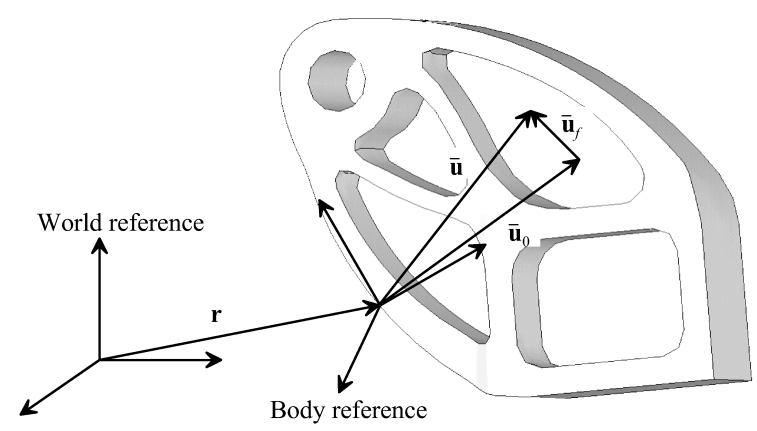

Figure 11: Floating reference frame.

In the case of simple geometries, such as beams [13], the set of data required to implement the flexible body dynamic equations, summarized in Table 1 , can be determined analytically, but in more general cases the use of finite elements (FE) computer codes as preprocessors is necessary. In this last case the huge number of nodal coordinates must be reduced to a much smaller number of modal coordinates, through the classical Craig-Bampton method [2] or other recently proposed methods $[17,18,14,19]$.

The Modelica model of a general flexible body: FEMBody, is characterized by an array of $N_{c}$ multibody connectors, while the data in Table 1 have been suitably collected in the Modelica record BodyData. The record is defined as replaceable:

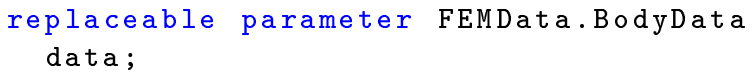

so that it is possible, by exploiting the features of the Modelica language, to assign a different data record to each FEMBody instance, by simply replacing the record in the model declaration:

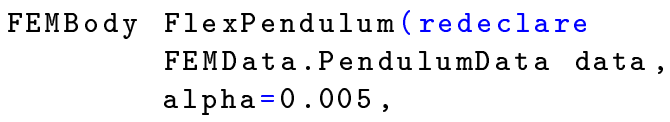


Table 1: Flexible body data.

\begin{tabular}{ll}
\hline$M$ & Number of deformation d.o.f. \\
$I^{1}, \mathbf{I}^{2}, \mathbf{I}_{i}^{3}, \mathbf{I}^{4}, \mathbf{I}_{i}^{5}, \mathbf{I}^{6}, \mathbf{I}^{7}, \mathbf{I}_{i}^{8}, \mathbf{I}_{i j}^{9}, \mathbf{I}_{i j}^{10}, \mathbf{I}_{i j}^{11}$ & Inertia invariants \\
$\mathbf{D}_{e}, \mathbf{K}_{e}$ & Structural damping and stiffness matrix \\
$N_{c}$ & Number of connectors \\
$\mathbf{S}_{i}, \hat{\mathbf{S}}_{i}$ & Slices of the modal matrix of connectors d.o.f. \\
$\overline{\mathbf{u}}_{0 i}, \overline{\mathbf{A}}_{i}$ & Undeformed position and orientation of connectors \\
\hline
\end{tabular}

beta $=0.005$,

$\mathrm{d}=1$ );

where alpha, beta, $d$ are the parameters defining the damping matrix (Rayleigh coefficients).

The process of generation of the flexible body data record is schematized in Fig. 12.

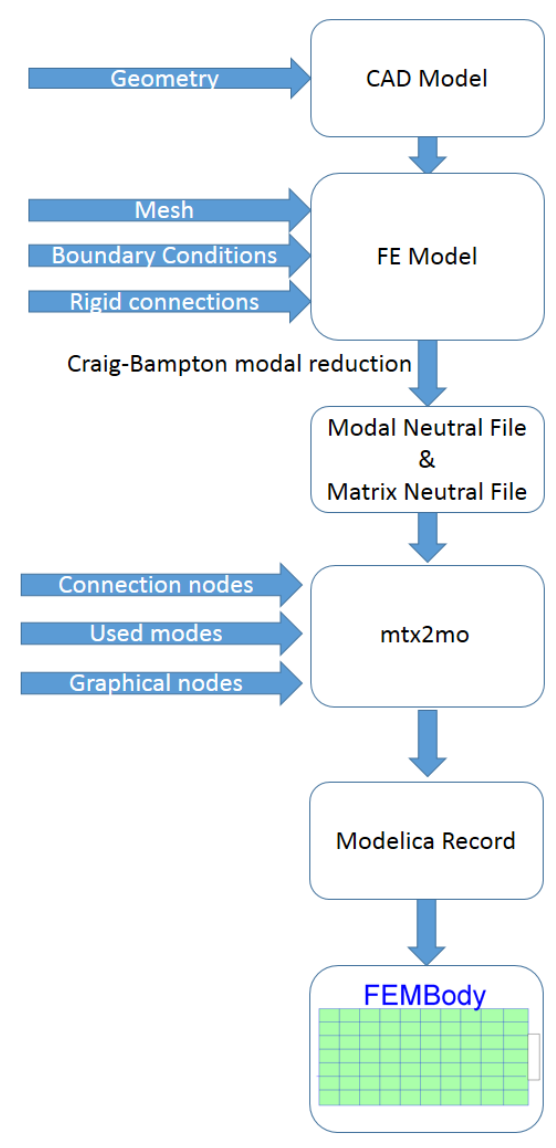

Figure 12: Flexible body data generation.

First, a FE model of the body is developed based on 3D CAD model (often inherited from design phase).

Then, a FEM analysis is performed, essentially consisting in an eigenfrequency analysis followed by a modal reduction step, generally based on the CraigBampton method.

The results of the FEM analysis are stored in a bi- nary Modal Neutral File (.mnf $)^{1}$, which must be then translated into an ASCII file, usually with extension . mtx, containing the same data in a readable format. This step can be performed through the Adams/Flex tool, a package included in the MSC.Adams suite, which allows to inspect the .mnf file and export the content in ASCII format.

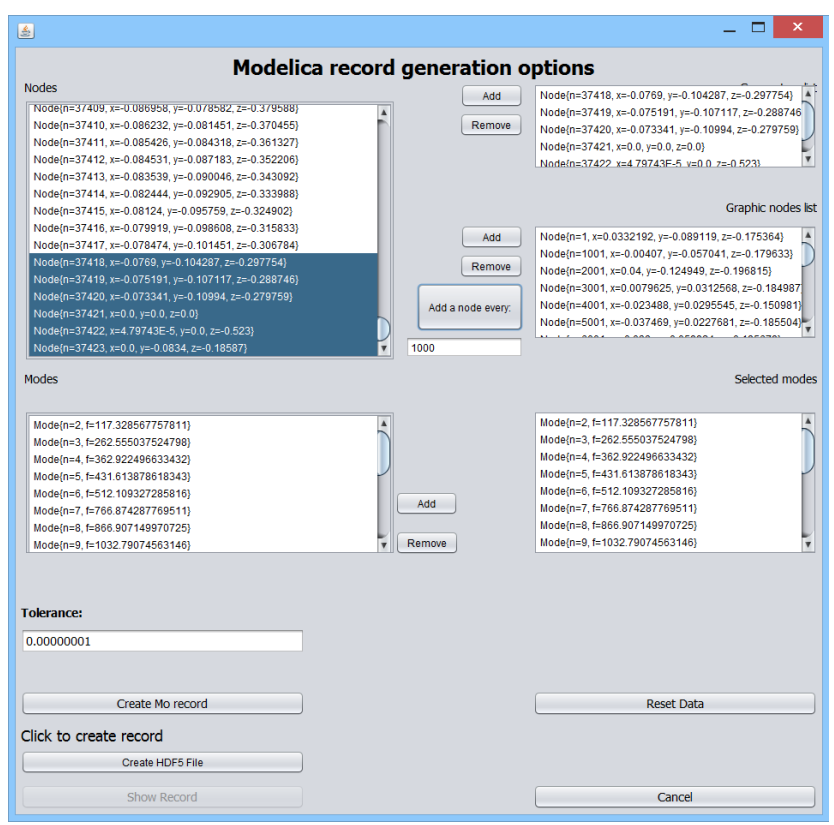

Figure 13: mtx2mo: Graphical User Interface.

The file containing the Modelica record of flexible body data is finally generated by a parsing software tool, named mtx $2 \mathrm{mo}$, written in $\mathrm{Java}^{2}$ (a screenshot of the tool is reported in Fig. 13). This tool reads the content of the . mtx file and translates it into the Modelica syntax, moreover, it allows the user to choose:

- the eigenmodes to be considered;

- which nodes of FE processing are selected as the position of the multibody connectors;

\footnotetext{
${ }^{1}$ The inertia invariants $\mathbf{I}^{10}$ and $\mathbf{I}^{11}$, are not a direct result of the analysis and are not stored in this file, they are computed by two Modelica functions from invariants $\mathbf{I}^{8}$ and $\mathbf{I}^{9}$, as detailed in [6].

${ }^{2}$ The tool could be implemented also in C or Modelica.
} 
- how many FE nodes are selected for the 3D graphic rendering of the model.

The described approach thus avoids the preprocessing stage adopted by the DLR FlexibleBodies library, which requires the models to be processed by FEMBS in order to obtain the SID file.

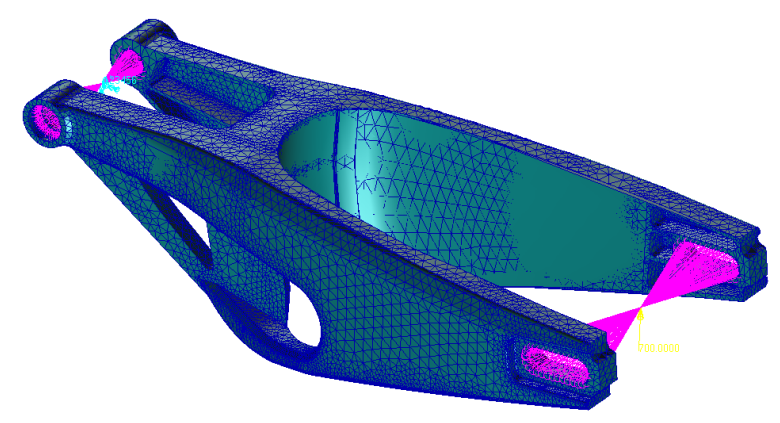

Figure 14: FE model of the swingarm.

\section{Modelling a flexible swingarm}

Figure 14 shows the FE model of the swingarm, realised with the Patran/Nastran suite by MSC.Software.

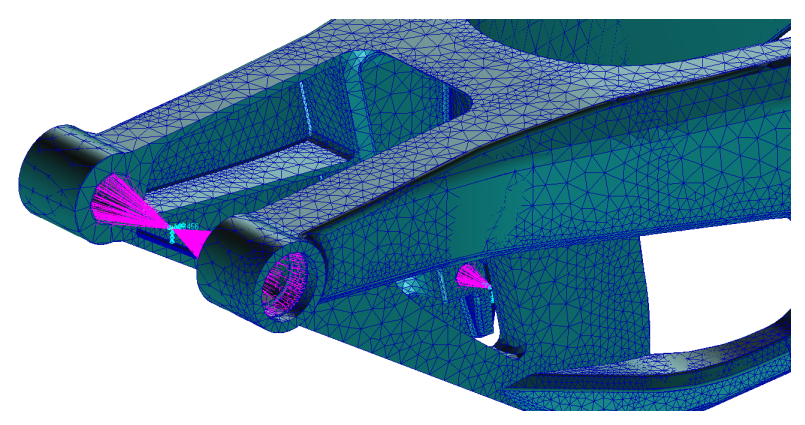

Figure 15: Multi-point constraint.

The swingarm is characterized by three connection points, so three virtual nodes have been defined in the FE geometry: one in the center of the frame pivot, one in the lower triangle pivot, and one in the center of the wheel hub. The internal surfaces of bores have been associated to this massless nodes by RBE2 rigid multipoint connections, namely, every node of the mesh located on the surface of the holes is rigidly connected to the virtual node, so that the motion of all dependent nodes is constrained by the motion of one node. Figure 15 shows the rigid connection between nodes in the front bore.

The boundary conditions were assigned in order to reproduce the hinge acting on the rear swingarm: all translations and two rotations were fixed for the pin connecting the swingarm to the mainframe, while all the other nodes were free to move. A free-motion eigenvalue resulted from the FEM analysis, with a very small absolute value $\left(1.2 \cdot 10^{-3} \mathrm{~Hz}\right)$, neglected in the generation of the Modelica model.

The first 20 eigenmodes were retained from the FEM analysis, with eigenfrequencies ranging from $117.3 \mathrm{~Hz}$ to $3585.6 \mathrm{~Hz}$, the first eigenmode is shown in Fig. 16.

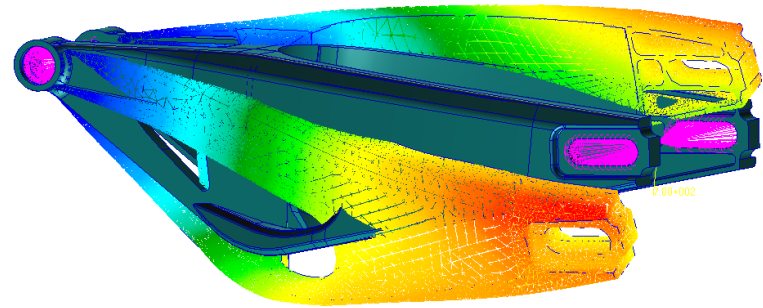

Figure 16: First torsional eigenmode.

It must be pointed out that structural damping is accounted in the flexible body model by means of the Rayleigh coefficients, which are difficult to estimate and are often the result of an averaging on the damping ratios of different modes. In this work the coefficients are chosen as $d=1, \alpha=\beta=0.005$.

The Modelica model of the flexible swingarm is shown in Fig. 17. Note that, for the sake of modularity, the connectors of the flexible body are stored in a vector, hence it is not possible to distinguish the connections in the graphical layer of the model.

It must be also pointed out that the RRR joint used in the rigid case to manage the kinematic loop is no longer required, as the flexibility of the body inherently breaks the loop.

A relative position sensor has been also introduced, with the aim of sensing the deflection of the rear wheel hub with respect to the position of the rear wheel bore in the undeformed configuration.

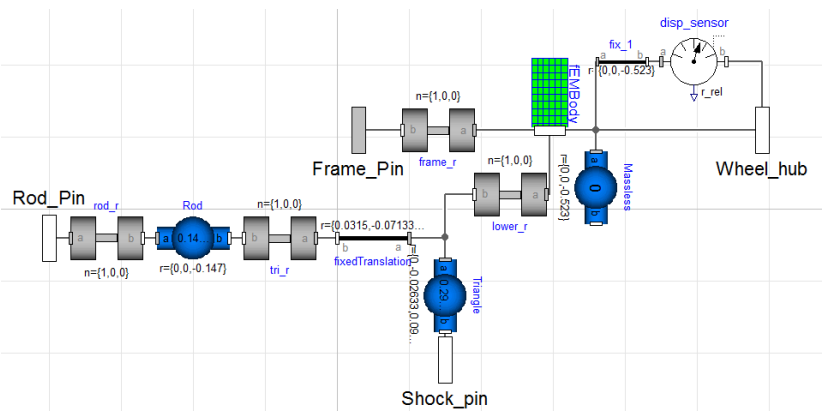

Figure 17: Modelica model of flexible swingarm. 


\section{Simulation results with a flexible swingarm}

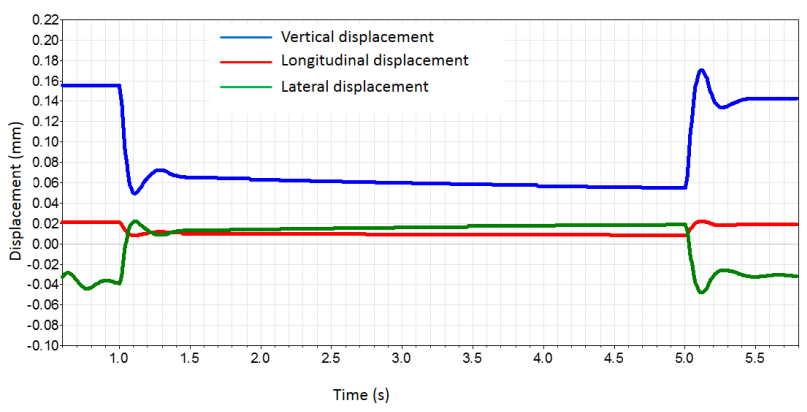

Figure 18: Displacement of rear wheel hub in braking.

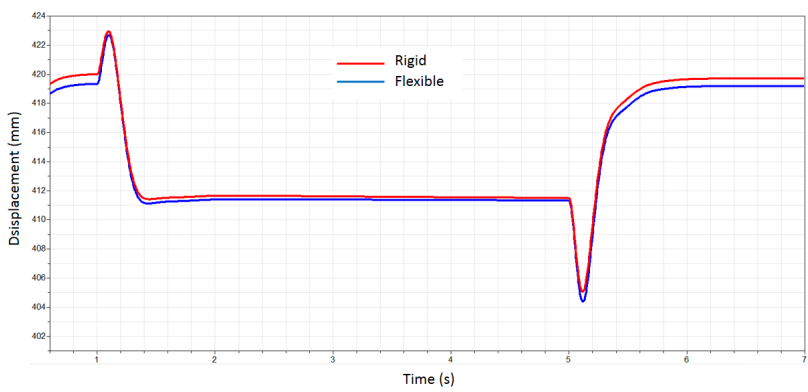

Figure 19: Vertical position of vehicle mass center during braking.

The experiments reported in Section 2 have been repeated with the flexible swingarm model.

Figure 18 shows the deflection of the rear hub measured by the above mentioned relative sensor. During the braking manoeuvre the swingarm deflects under the load on the rear part of the bike, starting from a value of $0.16 \mathrm{~mm}$ on the vertical axis before braking. A lateral displacement is also measured, due to the imprecise virtual driver, which cannot mantain the motorbike perfectly vertical. Although the swingarm deflection is small, it anyway affects the overall geometry of the vehichle.

In Fig. 19 the quotes of the mass center of the overall vechicle are shown, in the rigid and flexible case. During the braking, the weight and the inertia loads mainly impact on the front wheel, while the rear suspension reaches an equilibrium where poor forces are applied.

Forces and torques applied on the frame do not change significantly with respect to the rigid model, in Fig. 20 the vertical forces in the rear hinge are compared between the rigid and the flexible case at the beginning of the manoeuvre. As expected, the dif- ferences $(\approx 3 \%$ of the maximum value) are scarcely appreciable in the transients, and the deformation values are in good accordance with the static FE analysis (Fig. 21), in which the same loads (extracted from the multibody analysis) are applied.

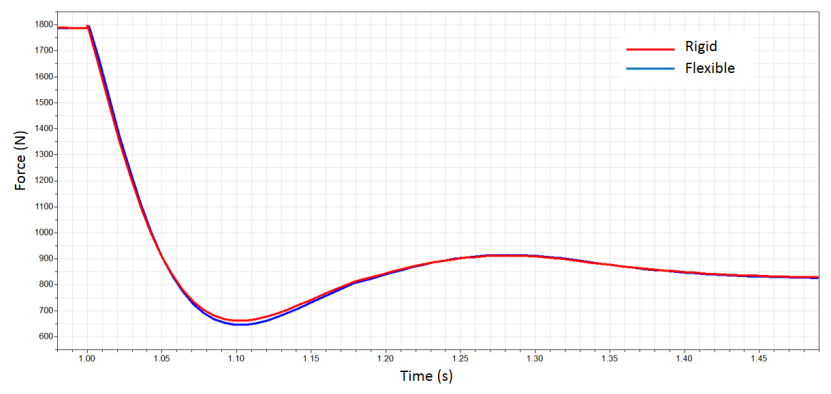

Figure 20: Vertical forces at the beginning of braking manoeuvre.

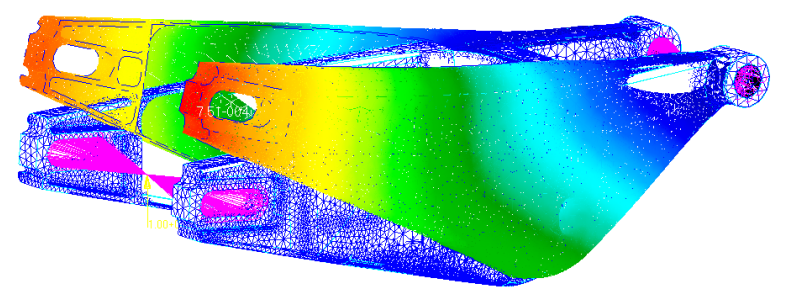

Figure 21: FEM static analisys of rear arm displacement.

During the simulated impacts with curbs the forces acting on the wheel, and consequently on the rear arm, are much higher in magnitude. Figure 22 shows the deflection of the rear wheel hub when the motorbike faces curbs. Note that in this case the vertical deflection reaches values up to $0.6 \mathrm{~mm}$.

Figure 23 shows a comparison between the vertical forces in the rear hinge in the rigid and flexible case. As expected, the forces in the flexible case are lower in absolute value, because part of the energy is used to deform the flexible component, which shows also a dissipative behaviour due to damping.

The overall behaviour of the motorbike in this case changes significantly due to flexibility, Fig.24 shows a comparison of the mass center quote of the motorbike in rigid and flexible case, the differences reach an absolute value of $10 \mathrm{~mm}$. Moreover, when considering the flexible swingarm, an interesting behaviour appears.

If the simulation time is long enough, the virtual driver is no more able to control the vehicle, which starts to wave after some seconds. Figure 25 shows 
the motorbike pose in both cases (flexible on the left and rigid on the right) at time $t=5.5 \mathrm{~s}$ : the vehicle with a flexible swingarm comes into an unstable behaviour and is going to thumble. This behaviour, currently under investigation, is certainly due to a poor performance of the virtual driver and to a rough model of the tyres, but appears to be induced by swingarm flexibility.

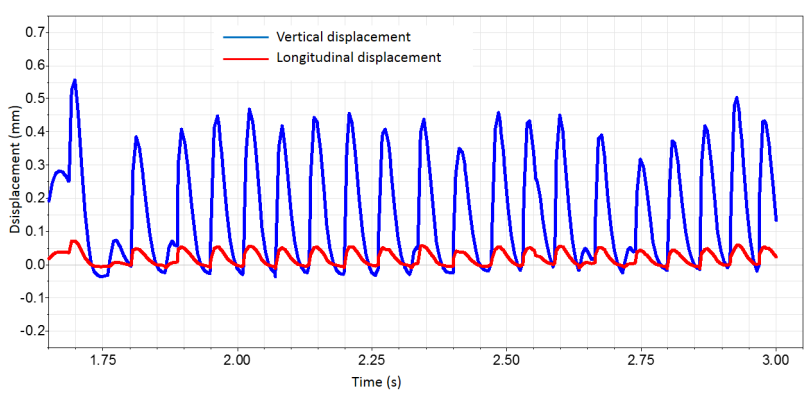

Figure 22: Vertical and longitudinal displacement of rear hub when facing curbs.

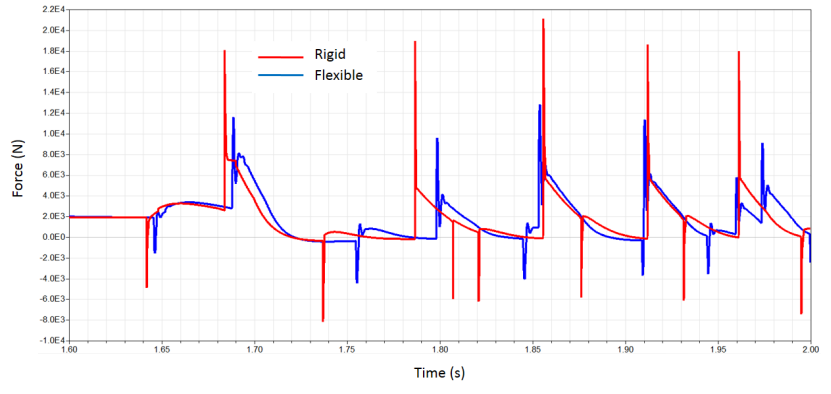

Figure 23: Vertical forces in rear hinge, comparison between rigid and flexible case.

The main drawback of model with the flexible component is the computational cost due to the additional elastic degrees of freedom: the braking simulation experiment with a rigid swingarm takes $0.32 \mathrm{~s}$ of CPU time to simulate $7 \mathrm{~s}$, on a normal laptop, the same model with a flexible swingarm takes about $10.4 \mathrm{sec}-$ onds of CPU time; regarding the simulation of impacts, the rigid model takes $5 \mathrm{~s}$ while the flexible model takes $64 \mathrm{~s}$ for $3.5 \mathrm{~s}$ of simulated time. The DASSL integration algorithm has been used in all the simulations.

\section{Conclusion and future work}

In this paper, a Modelica multibody model of a motorcycle with a flexible swingarm is presented.

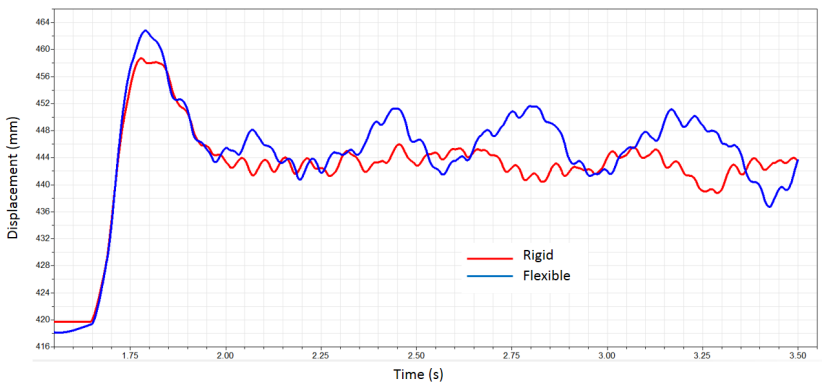

Figure 24: Mass center quote, comparison between rigid and flexible case.

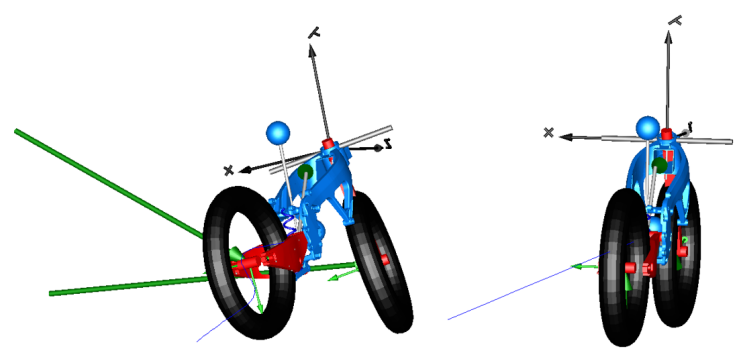

Figure 25: Motorbikes with rigid (right) and flexible (left) swingarm at time $t=5.5 \mathrm{~s}$.

At first, a rigid model of the motorbike has been developed and validated with respect to a sudden braking transient.

Then, a general approach to the modelling of flexible bodies is presented, and the full procedure leading to the Modelica model is detailed.

The proposed modelling approach has been applied to the rear swingarm of the motorbike and a comparison between the rigid and the flexible case is presented, with reference to a sudden braking and a series of impacts with curbs as simulation scenarios. In particular, the simulation of the motorbike with a flexible swingarm showed an unstable behaviour in the case of subsequent impacts, largely due to a poor performance of the virtual driver, but undoubtedly induced by swingarm flexibility.

The developed approach to flexible multibody modelling will allow to easily include the description of bodies' flexibility in mechatronic systems, expanding the range of the dynamic analysis. In particular, the said unstable behaviour is currently under investigation, as well as another unstable behaviour (shimmy) occurring in racing bikes. 


\section{Acknowledgements}

The authors would like to thank all people who supported this work: Dr. Marta Massera, for designing the frame and the swingarm, the Director of MUSP, prof. Michele Monno, and its staff for constant support, and RobbyMoto Engineering S.r.L., for valuable collaboration.

\section{References}

[1] G. Ferretti, G. Magnani, P. Rocco, Virtual prototyping of mechatronic systems, IFAC Journal Annual Reviews in Control 28 (2) (2004) 193206.

[2] R. R. Craig, M. C. C. Bampton, Coupling of substructures for dynamic analyses, AIAA Journal 6 (7) (1968) 1313-1319.

[3] A. Heckmann, M. Otter, S. Dietz, J. D. López, The DLR FlexibleBodies library to model large motions of beams and of exible bodies exported from nite element programs, in: $5^{\text {th }}$ Modelica Conference, Vienna, Austria, 2006, pp. 85-95.

[4] O. Wallrapp, Standardization of flexible body modeling in multibody system codes, Part I: Definition of Standard Input Data, Mechanics Based Design of Structures and Machines 22 (3) (1994) $283-304$.

[5] R. Schwertassek, O. Wallrapp, A. A. Shabana, Flexible multibody simulation and choice of shape functions, Nonlinear Dynamics 20 (1999) 361-380.

[6] G. Ferretti, A. Leva, B. Scaglioni, Objectoriented modelling of general flexible multibody systems, Mathematical and Computer Modelling of Dynamical Systems 20 (1) (2014) 1-22.

[7] A. A. Shabana, Dynamics of Multibody Systems, Cambridge University Press, 1998.

[8] F. Donida, G. Ferretti, S. M. Savaresi, M. Tanelli, Object-oriented modelling and simulation of a motorcycle, Mathematical and Computer Modelling of Dynamical Systems 14 (2) (2008) 79100.

[9] Marescotti, L., Modellazione dei sistema pilotaveicolo a due ruote in ambiente integrato MatlabAdams (in Italian), Master's thesis, Universiti $i^{1 / 2}$ di Pisa (2003).
[10] M. Otter, H. Elmqvist, S. Mattsson, The new Modelica multibody library, in: $3^{\text {rd }}$ Modelica Conference, Linköping, Sweden, 2003.

[11] G. Cocco, Dinamica e tecnica della motocicletta, 2013.

[12] W. Ritz, Über eine neue Methode zur Lösung gewisser Variationsprobleme der mathematischen Physik, Journal für die Reine und Angewandte Mathematik 135 (1909) 1-61.

[13] F. Schiavo, L. Viganò, G. Ferretti, Objectoriented modelling of flexible beams, Multibody System Dynamics 15 (3) (2006) 263 - 286.

[14] J. Fehr, P. Eberhard, Simulation process of flexible multibody systems with non-modal model order reduction techniques, Multibody System Dynamics 25 (2011) 313-334.

[15] U. Lugrís, M. A. Naya, A. Luaces, J. Cuadrado, Efficient calculation of the inertia terms in floating frame of reference formulations for flexible multibody dynamics, Proceedings of the Institution of Mechanical Engineers, Part K: Journal of Multi-body Dynamics 223 (2) (2009) 147-157.

[16] R. Schwertassek, O. Wallrapp, Dynamik flexibler Mehrkörpersysteme, Vieweg, Wiesbaden, 1999.

[17] P. Koutsovasilis, M. Beitelschmidt, Comparison of model reduction techniques for large mechanical systems, Multibody System Dynamics 20 (2) (2008) 111-128.

[18] M. Lehner, P. Eberhard, A two-step approach for model reduction in flexible multibody dynamics, Multibody System Dynamics 17 (2007) 157176.

[19] C. Nowakowski, J. Fehr, M. Fischer, P. Eberhard, Model order reduction in elastic multibody systems using the floating frame of reference formulation, in: $7^{\text {th }}$ Vienna International Conference on Mathematical Modelling - MATHMOD 2012, Vienna, Austria, 2012. 